\title{
One-pot synthesis of cinnamoyl hydrazides
}

\author{
Alan R. Katritzky,* Mingyi Wang, and Suoming Zhang§ \\ Department of Chemistry, Center for Heterocyclic Compounds, \\ University of Florida, Gainesville, FL 32611-7200 \\ E-mail: Katritzky@chem.ufl.edu
}

\section{Submitted to mark the $\mathbf{8 0}^{\text {th }}$ anniversary of Academician Mikhail Voronkov in acknowledgement of his distinguished contributions to chemistry \\ (received 10 Apr 01; accepted 25 Oct 01; published on the web 02 Nov 01)}

\begin{abstract}
An efficient one-pot conversion of cinnamic acids and heterocyclic analogs into the corresponding hydrazides in good to excellent yields under mild conditions involves hydrazinolysis of the intermediate $N$-acylbenzotriazoles.
\end{abstract}

Keywords: $N$-Acylbenzotriazole, cinnamoyl hydrazides, synthesis

\section{Introduction}

The synthesis of cinnamoyl hydrazides has attracted significant attention due to their utility as intermediates for the preparation of 1,2,4,5-tetrazines ${ }^{1}$ and their biological activity, e.g. as tuberculostats. ${ }^{2}$ Hydrazides are generally prepared by the hydrazinolysis of esters; ${ }^{3}$ however, cinnamic esters form pyrazolidones resulting from Michael addition as the main products. ${ }^{4 a, 4 b}$ Published routes to cinnamoyl hydrazides involve i) preliminary preparation of activated esters and/or amides using 1-hydroxybenzotriazole ( $\mathrm{HOBt}$ ) followed by reaction with a hydrazine, ${ }^{5}$ ii) treatment of activated esters ${ }^{4 b}$ or mixed anhydrides $1,{ }^{4 a}$ with hydrazine. As a continuation of our study on $N$-acylbenzotriazole chemistry, we describe herein an efficient procedure allowing for the direct conversion of cinnamic acids and heterocyclic analogs into hydrazides in excellent yields under mild conditions (Scheme 1).

\section{Results and Discussion}

In our earlier work, $N$-acylbenzotriazoles were used for formylation, ${ }^{6 \mathrm{a}}$ trifluoroacetylation, ${ }^{6 \mathrm{~b}}$ and for preparation of oxamides. ${ }^{6 c}$ Recent work has extended the applicability of acylbenzotriazoles 
as neutral acylating reagents to the preparation of primary, secondary and tertiary amides, ${ }^{7}$ although no $N$-acylbenzotriazoles derived from $\alpha, \beta$-unsaturated carboxylic acids were investigated in such reactions. Acylbenzotriazoles 2a-f can be prepared by the reaction of benzotriazole with acyl halides. ${ }^{8 \mathrm{a}, 8 \mathrm{~b}}$ However, our recently reported methodology ${ }^{7}$ provides a mild preparative method (Scheme 1), which can be applied to the synthesis of many molecules with sensitive groups. This technique was used to prepare 2a-f in excellent yields (Table 1). The structures of 2a-f were characterized on the basis of ${ }^{1} \mathrm{H}$ and ${ }^{13} \mathrm{C}$ NMR spectra and CHN analytical data.

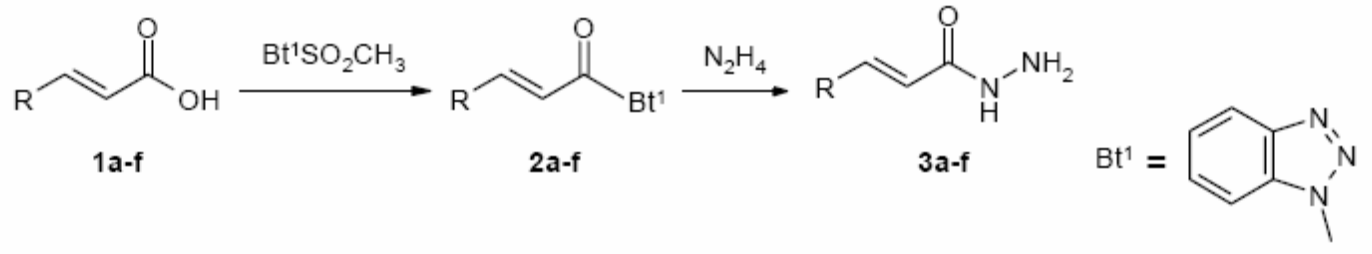

\section{Scheme 1}

Table 1. Preparation of $N$-acylbenzotriazoles 2a-f and hydrazides 3a-f ${ }^{\text {a }}$

\begin{tabular}{|c|c|c|c|c|c|c|}
\hline \multirow{2}{*}{ Entry } & \multirow{2}{*}{$\mathrm{R}$} & \multicolumn{2}{|c|}{$\mathrm{Mp}^{\mathrm{b}}\left[{ }^{\circ} \mathrm{C}\right]$} & \multicolumn{2}{|c|}{ Yield $^{\mathrm{b}}[\%]$} & \multirow{2}{*}{ Ref. } \\
\hline & & 2 & 3 & 2 & 3 & \\
\hline $\mathbf{a}$ & phenyl & $\begin{array}{c}157-159 \\
(158-159)\end{array}$ & $\begin{array}{c}116-117 \\
(116-117)\end{array}$ & $\begin{array}{c}90 \\
(90)\end{array}$ & $\begin{array}{c}85 \\
(66)\end{array}$ & $1,8 \mathrm{a}$ \\
\hline b & 4-chlorophenyl & $\begin{array}{c}183-184 \\
(191-192)\end{array}$ & $\begin{array}{c}154-155 \\
(154-157)\end{array}$ & $\begin{array}{c}96 \\
(88)\end{array}$ & $\begin{array}{l}86^{c} \\
(51)\end{array}$ & $2,8 b$ \\
\hline C & 4-nitrophenyl & $\begin{array}{l}242-243.5 \\
(244-245)\end{array}$ & $\begin{array}{c}210-212 \\
(215-216)\end{array}$ & $\begin{array}{c}91 \\
(90)\end{array}$ & $\begin{array}{c}78 \\
(70)\end{array}$ & $4 a, 8 b$ \\
\hline d & 3,4,5-trimethoxyphenyl & $136-137$ & $153-154$ & 98 & $83^{\mathrm{d}}$ & \\
\hline $\mathbf{e}$ & 2-furyl & $\begin{array}{c}142-144 \\
(143-144)\end{array}$ & $\begin{array}{c}93-94 \\
(108-109)\end{array}$ & $\begin{array}{c}95 \\
(58)\end{array}$ & $\begin{array}{c}81 \\
(69)\end{array}$ & $8 b, 9$ \\
\hline f & 2-thienyl & $169-170$ & $\begin{array}{c}108-110 \\
(123)\end{array}$ & 98 & 77 & 10 \\
\hline
\end{tabular}

${ }^{a}$ 2d, $2 \mathbf{f}$ and 3d are novel compounds. ${ }^{b}$ Data in parentheses are literature values. ${ }^{c}$ Yield by onepot synthesis is $81 \%$. ${ }^{\mathrm{d}}$ Yield by one-pot synthesis is $84 \%$.

Treatment of 2a-f with anhydrous hydrazine in THF gave 3a-f in more than $75 \%$ yields. Onepot synthesis, converting $\mathbf{1 b}, \mathbf{d}$ into $\mathbf{3 b}, \mathbf{d}$ by direct hydrazinolysis without isolation of $\mathbf{2} \mathbf{b}, \mathbf{d}$, also succeeded in rather good yields. Thus, hydrazinolysis in situ is a more effective strategy. Hydrazides 3a-f obtained are also summarized in Table 1. The benzotriazole byproduct formed in these reactions can be easily removed by washing with saturated aqueous $\mathrm{Na}_{2} \mathrm{CO}_{3}$.

In summary, we have developed an efficient general method to convert cinnamic acids and heterocyclic analogs into their hydrazides in one pot under mild conditions. 


\section{Experimental Section}

General Procedures. Melting points were determined on a MEL-TEMP capillary melting point apparatus equipped with a Fluke 51 digital thermometer. ${ }^{1} \mathrm{H}(300 \mathrm{MHz})$ and ${ }^{13} \mathrm{C}(75 \mathrm{MHz}) \mathrm{NMR}$ : spectra were recorded on a Varian GEMINI NMR spectrometer in $\mathrm{CDCl}_{3}$ unless stated otherwise, with TMS for ${ }^{1} \mathrm{H}$ and a solvent for ${ }^{13} \mathrm{C}$ as the internal standard. THF was distilled from sodium/ benzophenone under nitrogen immediately prior to use. All reactions with airsensitive compounds were carried out under argon atmosphere. Column chromatography was conducted with silica gel (230-400 mesh). 1-(Methylsulfonyl)benzotriazole was prepared according to the literature. ${ }^{7}$

General procedure for the preparation of $\boldsymbol{N}$-acylbenzotriazoles $2 \mathrm{a}-\mathbf{f}$. To a solution of the carboxylic acid 1 (10.0 mmol) and 1-(methylsulfonyl)benzotriazole $(1.97 \mathrm{~g}, 10.0 \mathrm{mmol})$ in of THF $(50 \mathrm{~mL})$, triethylamine $(2.0 \mathrm{~mL}, 14 \mathrm{mmol})$ was added dropwise at room temperature, and then the mixture was heated under reflux overnight. After the removal of the solvent and excess triethylamine under reduced pressure, the residue was dissolved in ethyl acetate $(150 \mathrm{~mL})$. The organic layer was washed with water, dried over anhydrous $\mathrm{MgSO}_{4}$, and evaporated. The resulting crude product was recrystallized from an appropriate solvent to provide the desired compounds $\mathbf{2 a}-\mathbf{f}$.

(E)-1-(Benzotriazol-1-yl)-3-phenyl-2-propen-1-one (2a). Cinnamic acid (1a) (1.48 g, 10 mmol) provided colorless needles 2a $(2.24 \mathrm{~g}, 90 \%)$, mp $157-159{ }^{\circ} \mathrm{C}$ (lit. $\left.{ }^{8 \mathrm{a}} 158-159{ }^{\circ} \mathrm{C}\right) ;{ }^{1} \mathrm{H}$ NMR: $\delta 8.41(\mathrm{~d}, J=8.2 \mathrm{~Hz}, 1 \mathrm{H}), 8.16-8.13(\mathrm{~m}, 3 \mathrm{H}), 7.76-7.73(\mathrm{~m}, 2 \mathrm{H}), 7.67(\mathrm{dd}, J=8.0,7.3$ $\mathrm{Hz}, 1 \mathrm{H}), 7.53(\mathrm{dd}, J=8.0,7.4 \mathrm{~Hz}, 1 \mathrm{H}), 7.48-7.46(\mathrm{~m}, 3 \mathrm{H}) ;{ }^{13} \mathrm{C} \mathrm{NMR}: \delta 163.9,148.7,146.3$, $134.0,131.4,130.2,129.0,128.9,127.7,126.2,120.1,115.9,114.8$.

(E)-1-(Benzotriazol-1-yl)-3-(4-chlorophenyl)-2-propen-1-one (2b). From 4-chlorocinnamic acid (1b) (1.82 g, $10 \mathrm{mmol})$ colorless needles $2 \mathbf{b}(2.72 \mathrm{~g}, 96 \%)$ were obtained, mp $183-184{ }^{\circ} \mathrm{C}$ (lit. $\left.{ }^{8 \mathrm{~b}} 191-192{ }^{\circ} \mathrm{C}\right) ;{ }^{1} \mathrm{H}$ NMR: $\delta 8.40(\mathrm{~d}, J=8.2 \mathrm{~Hz}, 1 \mathrm{H}), 8.14(\mathrm{~d}, J=8.5 \mathrm{~Hz}, 1 \mathrm{H}), 8.11(\mathrm{~d}, J=$ $16.4 \mathrm{~Hz}, 1 \mathrm{H}), 8.05(\mathrm{~d}, J=16.4 \mathrm{~Hz}, 1 \mathrm{H}), 7.68-7.66(\mathrm{~m}, 3 \mathrm{H}), 7.53(\mathrm{dd}, J=7.9,7.3 \mathrm{~Hz}, 1 \mathrm{H}), 7.43$ $(\mathrm{d}, J=8.5 \mathrm{~Hz}, 2 \mathrm{H}) ;{ }^{13} \mathrm{C} \mathrm{NMR}: \delta 163.6,147.0,146.3,137.4,132.5,131.4,130.3,130.0,129.4$, $126.3,120.2,116.6,114.7$.

(E)-1-(Benzotriazol-1-yl)-3-(4-nitrophenyl)-2-propen-1-one (2c). 4-Nitrocinnamic acid (1c) $(1.93 \mathrm{~g}, 10 \mathrm{mmol})$ afforded yellow microcrystals $2 \mathrm{c}(2.68 \mathrm{~g}, 90 \%), \mathrm{mp} 242-243.5{ }^{\circ} \mathrm{C}$ (lit. ${ }^{8 \mathrm{~b}}$ 244-245 $\left.{ }^{\circ} \mathrm{C}\right) ;{ }^{1} \mathrm{H}$ NMR: $\delta 8.42(\mathrm{~d}, J=8.2 \mathrm{~Hz}, 1 \mathrm{H}), 8.33(\mathrm{~d}, J=8.0 \mathrm{~Hz}, 2 \mathrm{H}), 8.26(\mathrm{~d}, J=16.1 \mathrm{~Hz}$, $1 \mathrm{H}), 8.17(\mathrm{~d}, J=5.3 \mathrm{~Hz}, 1 \mathrm{H}), 8.16(\mathrm{~d}, J=16.1 \mathrm{~Hz}, 1 \mathrm{H}), 7.91(\mathrm{~d}, J=8.1 \mathrm{~Hz}, 2 \mathrm{H}), 7.73(\mathrm{dd}, J=$ 8.1, 7.4 Hz, 1H), $7.57(\mathrm{dd}, J=8.0,7.5 \mathrm{~Hz}, 1 \mathrm{H}) ;{ }^{13} \mathrm{C} \mathrm{NMR}: \delta 163.0,149.0,146.4,145.2,139.9$, $131.3,130.6,129.5,126.6,124.3,120.4,120.3,114.7$.

(E)-1-(Benzotriazol-1-yl)-3-(3,4,5-trimethoxyphenyl)-2-propen-1-one (2d). 3,4,5-Trimethoxy cinnamic acid (1d) $(2.38 \mathrm{~g}, 10 \mathrm{mmol})$ gave yellow needles $2 \mathbf{d}\left(3.32 \mathrm{~g} \mathrm{98 \% ),} \mathrm{mp} \mathrm{136-137}{ }^{\circ} \mathrm{C} ;{ }^{1} \mathrm{H}\right.$ NMR: $\delta 8.29(\mathrm{~d}, J=8.2 \mathrm{~Hz}, 1 \mathrm{H}), 8.07(\mathrm{~d}, J=8.2 \mathrm{~Hz}, 1 \mathrm{H}), 7.95(\mathrm{~d}, J=15.7 \mathrm{~Hz}, 1 \mathrm{H}), 7.88(\mathrm{~d}, J=$ 
$15.7 \mathrm{~Hz}, 1 \mathrm{H}), 7.60(\mathrm{t}, J=7.6 \mathrm{~Hz}, 1 \mathrm{H}), 7.46(\mathrm{dd}, J=7.6,7.3 \mathrm{~Hz}, 1 \mathrm{H}), 6.87(\mathrm{~s}, 2 \mathrm{H}), 3.93(\mathrm{~s}, 6 \mathrm{H})$, 3.92 (s, 3H); ${ }^{13} \mathrm{C}$ NMR: $\delta$ 163.2, 153.1, 148.2, 145.9, 140.9, 131.0, 129.7, 129.0, 125.7, 119.7, 114.5, 114.4, 105.9, 60.6, 55.9. Anal. Calcd for $\mathrm{C}_{18} \mathrm{H}_{17} \mathrm{~N}_{3} \mathrm{O}_{4}$ (339.35): C, 63.71; H, 5.05; N, 12.38. Found: C, 63.37; H, 5.37; N, 11.93.

(E)-1-(Benzotriazol-1-yl)-3-(2-furyl)-2-propen-1-one (2e). 3-(2-Furyl)acrylic acid 1e, (1.38 g, $10 \mathrm{mmol})$ gave dark-red needles $2 \mathbf{e}(2.27 \mathrm{~g}, 95 \%)$, mp $142-144{ }^{\circ} \mathrm{C}\left(\mathrm{lit}^{.8 \mathrm{~b}} 143-144{ }^{\circ} \mathrm{C}\right)$; ${ }^{1} \mathrm{H}$ NMR: $\delta 8.37(\mathrm{~d}, J=8.2 \mathrm{~Hz} .1 \mathrm{H}), 8.12(\mathrm{~d}, J=8.2 \mathrm{~Hz}, 1 \mathrm{H}), 7.94(\mathrm{~d}, J=15.5 \mathrm{~Hz}, 1 \mathrm{H}), 7.84(\mathrm{~d}, J=15.7$ $\mathrm{Hz}, 1 \mathrm{H}), 7.67-7.60(\mathrm{~m}, 2 \mathrm{H}), 7.49(\mathrm{dd}, J=7.9,7.3 \mathrm{~Hz}, 1 \mathrm{H}), 6.84(\mathrm{~d}, J=3.2 \mathrm{~Hz}, 1 \mathrm{H}), 6.55$ (br s, $1 \mathrm{H}) ;{ }^{13} \mathrm{C}$ NMR: $\delta 163.8,151.0,146.2,145.9,133.9,131.3,130.0,125.9,120.0,117.5,114.6$, $113.4,112.8$.

(E)-1-(Benzotriazol-1-yl)-3-(2-thienyl)-2-propen-1-one (2f). 3-(2-Thienyl)acrylic acid (1f) (1.54 g, $10 \mathrm{mmol})$ afforded yellow needles $2 \mathrm{f}(2.50 \mathrm{~g}, 98 \%), \mathrm{mp} 169-170{ }^{\circ} \mathrm{C} ;{ }^{1} \mathrm{H}$ NMR: $\delta 8.40$ $(\mathrm{d}, J=8.2 \mathrm{~Hz}, 1 \mathrm{H}), 8.24(\mathrm{~d}, J=15.5 \mathrm{~Hz}, 1 \mathrm{H}), 8.14(\mathrm{~d}, J=8.2 \mathrm{~Hz}, 1 \mathrm{H}), 7.88(\mathrm{~d}, J=15.5 \mathrm{~Hz}, 1 \mathrm{H})$, $7.67(\mathrm{dd}, J=7.9,7.6 \mathrm{~Hz}, 1 \mathrm{H}), 7.54-7.43(\mathrm{~m}, 3 \mathrm{H}), 7.13(\mathrm{dd}, J=4.4,4.1 \mathrm{~Hz}, 1 \mathrm{H}) ;{ }^{13} \mathrm{C} \mathrm{NMR}: \delta$ 163.7, 146.2, 140.7, 139.6, 133.0, 131.4, 130.5, 130.2, 128.4, 126.1, 120.1, 114.7, 114.4. Anal. Calcd for $\mathrm{C}_{13} \mathrm{H}_{9} \mathrm{~N}_{3} \mathrm{OS}$ (255.30): C, 61.16; H, 3.55; N, 16.46. Found: C, 61.27; H, 3.32; N, 16.47.

\section{General procedure for the preparation of hydrazides 3a-f}

To a solution of the appropriate $N$-acylbenzotriazoles $2(10 \mathrm{mmol})$ in THF $(50 \mathrm{~mL})$, a solution of anhydrous hydrazine $(0.38 \mathrm{~mL}, 12 \mathrm{mmol})$ in THF $(5 \mathrm{~mL})$ was added dropwise at room temperature. The reaction mixture was stirred at room temperature for about $4 \mathrm{~h}$. After THF was removed under reduced pressure, the residue was dissolved in ethyl acetate $(150 \mathrm{~mL})$. The organic layer was washed with saturated aqueous $\mathrm{Na}_{2} \mathrm{CO}_{3}$, water, dried over anhydrous $\mathrm{MgSO}_{4}$, and evaporated to give a crude product, which was recrystallized from benzene.

(E)-3-Phenyl-2-propenohydrazide (3a). From 2a (2.49 g, $10 \mathrm{mmol})$ were obtained white plates 3a $(1.38 \mathrm{~g}, 85 \%), \mathrm{mp} 116-117{ }^{\circ} \mathrm{C}\left(\operatorname{lit}^{1} 116-117{ }^{\circ} \mathrm{C}\right) ;{ }^{1} \mathrm{H}$ NMR: $\delta 7.68(\mathrm{~d}, J=15.7 \mathrm{~Hz}, 1 \mathrm{H})$, 7.48-7.51 (m, 2H), 7.40-7.43 (m, 1H), 7.34-7.39 (m, 3H), $6.40(\mathrm{~d}, J=15.7 \mathrm{~Hz}, 1 \mathrm{H}), 3.95-4.11$ (br s, $2 \mathrm{H}) ;{ }^{13} \mathrm{C}$ NMR: $\delta 167.1,141.3,134.5,129.7,128.7,127.7,118.1$.

(E)-3-(4-Chlorophenyl)-2-propenohydrazide (3b). 2b (2.84 g, $10 \mathrm{mmol})$ afforded white plates 2b (1.69 g, 86\%), mp 154-155 ${ }^{\circ} \mathrm{C}$ (lit. $\left.{ }^{2} 154-157^{\circ} \mathrm{C}\right) ;{ }^{1} \mathrm{H}$ NMR: $\delta 7.64$ (d, $\left.J=15.5 \mathrm{~Hz}, 1 \mathrm{H}\right), 7.43$ $(\mathrm{d}, J=8.5 \mathrm{~Hz}, 2 \mathrm{H}), 7.34(\mathrm{~d}, J=8.5 \mathrm{~Hz}, 2 \mathrm{H}), 7.26(\mathrm{br} \mathrm{s}, 1 \mathrm{H}), 6.33(\mathrm{~d}, J=15.5 \mathrm{~Hz}, 1 \mathrm{H}), 4.08$ (br s, $2 \mathrm{H}) ;{ }^{13} \mathrm{C}$ NMR: $\delta 166.7,140.5,135.8,132.9,129.1,128.9,118.2$.

(E)-3-(4-Nitrophenyl)-2-propenohydrazide (3c). 2c (2.94 g, $10 \mathrm{mmol})$ provided white plates 3c (1.61 g, 78\%), mp 210-212 ${ }^{\circ} \mathrm{C}$ (lit. ${ }^{4 \mathrm{a}} 215-216^{\circ} \mathrm{C}$ ); ${ }^{1} \mathrm{H}$ NMR: (DMSO-d $) \delta 9.55(\mathrm{~s}, 1 \mathrm{H}), 8.26$ $(\mathrm{d}, J=8.8 \mathrm{~Hz}, 2 \mathrm{H}), 7.83(\mathrm{~d}, J=8.8 \mathrm{~Hz}, 2 \mathrm{H}), 7.56(\mathrm{~d}, J=15.9 \mathrm{~Hz}, 1 \mathrm{H}), 6.73(\mathrm{~d}, J=15.9 \mathrm{~Hz}, 1 \mathrm{H})$, 4.56 (br s, $2 \mathrm{H}$ ); ${ }^{13} \mathrm{C}$ NMR: (DMSO-d 6 ) $\delta 163.5,147.5,141.6,135.9,128.5,124.6,124.2$.

(E)-3-(3,4,5-Trimethoxyphenyl)-2-propenohydrazide (3d). 2d (3.39 g, $10 \mathrm{mmol})$ gave white needles 3d (2.10 g, 83\%), mp 153-154 ${ }^{\circ} \mathrm{C} ;{ }^{1} \mathrm{H}$ NMR: $\delta 7.60(\mathrm{~d}, J=15.4 \mathrm{~Hz}, 1 \mathrm{H}), 7.22$ (br s, 1H), $6.73(\mathrm{~s}, 2 \mathrm{H}), 6.33$ (d, $J=15.5 \mathrm{~Hz}, 1 \mathrm{H}), 4.10$ (br s, 1H), $3.87(\mathrm{~s}, 9 \mathrm{H}) ;{ }^{13} \mathrm{C}$ NMR: $\delta 166.9,153.3$, 141.6, 139.7, 130.0, 117.1, 104.9, 60.9, 56.1. Anal. Calcd for $\mathrm{C}_{12} \mathrm{H}_{16} \mathrm{~N}_{2} \mathrm{O}_{4}$ (252.27): C, 57.13; $\mathrm{H}$, 
6.39; N, 11.10. Found: C, 57.25; H, 6.75; N, 11.05 .

(E)-3-(2-Furyl)-2-propenohydrazide (3e). From 2e $(2.39 \mathrm{~g}, 10 \mathrm{mmol})$ were obtained white needles 3e (1.23 g, 81\%), mp 93-94 ${ }^{\circ} \mathrm{C}$ (lit. $\left.{ }^{9} 108-110{ }^{\circ} \mathrm{C}\right) ;{ }^{1} \mathrm{H}$ NMR: $\delta 7.46(\mathrm{~d}, J=15.2 \mathrm{~Hz}, 1 \mathrm{H})$, 7.43 (s, 1H), 7.36 (br s, 1H), 6.56 (d, $J=2.9 \mathrm{~Hz}, 1 \mathrm{H}), 6.45$ (d, $J=1.5 \mathrm{~Hz}, 1 \mathrm{H}), 6.30$ (d, $J=15.2$ $\mathrm{Hz}, 1 \mathrm{H}$ ), 4.06 (br s, $2 \mathrm{H}) ;{ }^{13} \mathrm{C}$ NMR: $\delta 167.0,150.9$, 144.1, 128.3, 115.5, 114.2, 112.1. Anal. Calcd for $\mathrm{C}_{7} \mathrm{H}_{8} \mathrm{~N}_{2} \mathrm{O}_{2}$ (152.15): C, 55.25; H, 5.30; N, 18.41. Found: C, 55.49; H, 4.93; N, 18.25.

(E)-3-(2-Thienyl)-2-propenohydrazide (3f). $2 \mathbf{f}(2.55 \mathrm{~g}, 10 \mathrm{mmol})$ provided white needles $3 f$ (1.30 g, 77\%), mp 108-110 ${ }^{\circ} \mathrm{C}$ (lit. $\left.{ }^{10} 123{ }^{\circ} \mathrm{C}\right) ;{ }^{1} \mathrm{H}$ NMR: $\delta 7.81(\mathrm{~d}, J=15.5 \mathrm{~Hz}, 1 \mathrm{H}), 7.47$ (s, $1 \mathrm{H}), 7.32(\mathrm{~d}, J=5.0 \mathrm{~Hz}, 1 \mathrm{H}), 7.21(\mathrm{~d}, J=2.0 \mathrm{~Hz}, 1 \mathrm{H}), 7.03(\mathrm{~d}, J=3.5 \mathrm{~Hz}, 1 \mathrm{H}), 6.21(\mathrm{~d}, J=15.5$ $\mathrm{Hz}, 1 \mathrm{H}$ ), 4.10 (br s, $2 \mathrm{H}$ ); ${ }^{13} \mathrm{C}$ NMR: $\delta 166.9$, 139.6, 134.2, 130.6, 127.9, 127.5, 116.6. Anal. Calcd for $\mathrm{C}_{7} \mathrm{H}_{8} \mathrm{~N}_{2} \mathrm{OS}$ (168.22): C, 49.98; H, 4.79; N, 16.65. Found: C, 50.14; H, 4.65; N, 16.59.

\section{References and Notes}

§ Current address: Neurogen Corporation, 35 N.E. Industrial Road, Branford, CT 06405, USA.

1. Lifka, T.; Meier, H. J. Prakt. Chem. 1995, 337, 641.

2. Anzai, K; Suzuki, S. J. Antibiotics Ser. A 1962, 15, 202.

3. Wagner, R. B.; Zook, H. D. Synthetic Organic Chemistry, John Wiley \& Sons: New York, 1953 ; p 569.

4. (a) Godtfredsen, W. O.; Vangedal, S. Acta Chem. Scand. 1955, 9, 1498. (b) Harada, R.; Kondo, H. Bull. Chem. Soc. Jpn. 1968, 41, 2521.

5. Zhang, X.; Patel, M.; Maryanoff, C. A.; Sorgi, K. L. 220th ACS Natl. Meeting 2000, Abstr. ORGN-158.

6. (a) Katritzky, A. R.; Chang, H.-X.; Yang, B. Synthesis 1995, 503. (b) Katritzky, A. R.; Yang, B.; Semenzin, D. J. Org. Chem. 1997, 62, 726. (c) Katritzky, A. R.; Levell, J. R.; Pleynet, D. P. M. Synthesis 1998, 153.

7. Katritzky, A. R.; He, H.-Y.; Suzuki, K. J. Org. Chem. 2000, 65, 8210.

8. (a) Kreutzberger, A.; Dietz, E. Tetrahedron Lett. 1970, 1457. (b) Kreutzberger, A.; van der Goot, H. J. Heterocycl. Chem. 1975, 12, 665.

9. Yale, H. L.; Losee, K.; Martins, J.; Holsing, M; Perry, F. M.; Bernstein, J. J. Am. Chem. Soc. 1953, 75, 1933.

10. Maziere, B.; Maziere, M.; Bavay, J. C.; Nguyen, D. X. Chim. Ther. 1969, 4, 265. 